\title{
Gradhiva
}

GRADHI

Revue d'anthropologie et d'histoire des arts

$2 \mid 2005$

Autour de Lucien Sebag

\section{Par Marie-Laure Bernadac}

\section{Marie-Laure Bernadac}

\section{OpenEdition}

Journals

Édition électronique

URL : http://journals.openedition.org/gradhiva/525

DOI : 10.4000/gradhiva.525

ISSN : 1760-849X

\section{Éditeur}

Musée du quai Branly Jacques Chirac

\section{Édition imprimée}

Date de publication : 1 novembre 2005

Pagination : 145-146

ISBN : 2-915-133-10-7

ISSN : 0764-8928

Référence électronique

Marie-Laure Bernadac, "Par Marie-Laure Bernadac », Gradhiva [En ligne], 2 | 2005, mis en ligne le 10 décembre 2008, consulté le 15 septembre 2020. URL : http://journals.openedition.org/gradhiva/525

Ce document a été généré automatiquement le 15 septembre 2020.

(C) musée du quai Branly 


\title{
Par Marie-Laure Bernadac
}

\author{
Marie-Laure Bernadac
}

\section{NOTE DE L'AUTEUR}

Le catalogue de l'exposition a été édité sous la direction de Marie-Laure Bernadac.

Paris, centre Georges-Pompidou, 2005, ill. noir et blanc et couleurs, 350 p.

1 La manipulation des textes est hélas un fait trop courant dans la presse pour être relevée, mais lorsque cette pratique va de pair avec un contresens majeur sur les enjeux d'un projet artistique, il est alors nécessaire d'intervenir.

2 Les extraits cités par l'auteur des textes d'introduction ont été détournés de leur contexte. Quand j'emploie le terme d'aventure, non pour définir l'exposition, mais pour parler de la reconnaissance récente de la création contemporaine africaine longtemps méconnue, ce n'est évidemment pas pour revenir à une conception ethnographique d'un autre temps. C'est pour évoquer le caractère hasardeux, aventureux du domaine dans lequel on travaille, dans la mesure où il n'y a pas encore de répères ni de théorie pour étayer les choix artistiques et définir des concepts. Et quand je dénonce l'esprit pionnier qui caractérise les points de vue de certains spécialistes, c'est également pour stigmatiser les a priori de certains des acteurs de ce nouveau champ, qui n'ont qu'une vue partielle de ce que l'on entend par art africain et par création contemporaine.

3 Tant Simon Njami que moi-même avons clairement insisté sur le fait qu'Africa Remix ne devait rien à l'approche anthropologique et ne relevait pas d'une conception essentialiste de l'art africain. Il s'agit au contraire, par le choix des artistes présentés et des œuvres sélectionnées, de sortir enfin les artistes africains de cette vision primitiviste. Même si nous devons reconnaître que certaines œuvres et certains artistes pourraient faire l'objet de cette critique. Plutôt que des généralités, on aurait aimé lire une analyse précise de ces œuvres, une définition de leur caractère " primitif ", et poser alors la question de leur légitimité dans cette exposition. Cette critique, par ailleurs, est paradoxale, car dans le même temps on nous reproche de ne pas " contextualiser » les œuvres, ce qui reviendrait alors à une approche anthropologique. 
4 De plus, je ne vois pas en quoi les termes d'identité et d'histoire relèveraient uniquement de l'anthropologie. C'est faire preuve d'une méconnaissance grave de la création artistique moderne et contemporaine, dont tout un pan - depuis Marcel Duchamp en passant par les femmes artistes et, aujourd'hui, les artistes de la diaspora - prend justement comme thématique celle de l'identité. Il en va de même pour l'histoire, la ville et le corps. Ces thèmes universaux, sous des appellations diverses, se retrouvent depuis une dizaine d'années dans nombre de biennales et d'expositions internationales sans que cela soulève ce type de critique stérile.

5 Cette critique de l'exposition, qui n'aborde pratiquement aucune œuvre et aucun artiste, est symptomatique du malentendu qui a été entretenu par quelques personnes sur ce projet. Ils partent généralement de deux postulats de base :

1. on ne peut plus faire à notre époque une exposition collective à caractère national ou géographique, car les artistes sont aujourd'hui nomades et l'art est au-delà des frontières ;

2. on ne peut pas exposer l'art africain sans tenir compte des théories postcoloniales.

6 Ces arguments me paraissent un peu courts et surtout hypocrites. D'une part, on sait très bien qu'ils ne sont pas employés à l'égard des autres nations ni des autres continents ${ }^{1}$. D'autre part, une exposition collective n'est pas la mise en application d'un concept ou d'une théorie ${ }^{2}$, elle est, aussi et surtout, une façon de produire des œuvres nouvelles, de faire connaître des artistes encore peu reconnus sur la scène internationale malgré leur talent, et de permettre de susciter un vrai débat sur les questions posées par les artistes et par les œuvres présentées.

7 Des critiques pouvaient être faites à cette exposition (choix de certains artistes, absence d'autres, œuvres déjà vues, etc.). Je suis parfois moi-même la première à les faire, mais se débarrasser du sujet de façon aussi rapide, sans prendre en compte la création des artistes, ni citer leurs noms, ni analyser leur travail, non seulement ne fait pas beaucoup avancer la question, mais nous ramène plus de quinze ans en arrière.

8 Aborder le domaine de l'Afrique relève en effet de la quadrature du cercle. Il faudrait pouvoir dans le même temps considérer les artistes originaires d'Afrique comme des artistes internationaux indépendamment du contexte de production, de diffusion, des thématiques de leurs œuvres, tout en tenant compte de ces mêmes paramètres afin de ne pas uniformiser l'art contemporain mondial. Toute approche est considérée soit comme trop exotique, trop ethnographique ou pas assez, et jamais les œuvres ne sont analysées en tant que telles.

9 Pourquoi ne pourrait-on pas en effet montrer dans la même exposition un artiste comme Frédéric Bruly Bouabré et une jeune artiste issue d'une école d'art parisienne? On présente bien à certaines occasions (Documenta, Venise, etc.) une artiste comme Louise Bourgeois avec de jeunes artistes issus d'une culture et d'une formation totalement différentes.

Quant aux critiques sur l'accrochage, je concède bien volontiers la spécificité exceptionnelle de la présentation de la peinture de Cyprien Toukoudagba ${ }^{3}$, nécessitée par le désir de présenter une œuvre reproduite dans le catalogue, prêtée de plus par le musée du quai Branly. Mais par ailleurs l'architecte du centre Pompidou a dessiné deux larges voies de circulation qui permettaient, me semble-t-il, d'avoir une bonne visibilité des œuvres présentées et de réserver des espaces spécifiques pour les installations. L'accrochage répondait à une logique tant thématique que formelle, très ordonnée, presque trop pour certains, et n'avait rien d'une cacophonie. 
11 Je relèverai enfin une dernière imprécision. Si un certain nombre d'œuvres avait déjà été montrées à l'étranger, une dizaine d'autres ont été produites spécialement pour l'exposition, et pas seulement le salon de Cheick Diallo, comme l'écrit l'auteur.

12 J'ajouterai enfin que l'exposition, malgré ses imperfections, a rencontré à Paris un large succès, tant auprès du public (plus de 150000 visiteurs en deux mois et 10 jours) que de la presse. Elle circulera en 2006 aux Canaries, au Japon, en Suède, avant d'être présentée en 2007 en Afrique du Sud.

13 Autant de facteurs positifs qui permettront sans doute une meilleure connaissance de ces artistes et favoriseront, je l'espère, une autre lecture de l'histoire de l'art.

\section{NOTES}

1. Voir par exemple les critiques récentes concernant tant l'art chinois que brésilien, ou même les polémiques sur l'art français.

2. On s'aperçoit que la plupart des expositions citées comme exemplaires, (Authentic/Excentric, Short Century, Fault Lines) n'ont pas été vues, seulement perçues par le catalogue, et qu'elles présentent la plupart du temps les mêmes artistes qu'Africa Remix.

3. Dans la mesure où ces peintures à l'origine décoraient directement les murs des maisons ou des temples, il ne serait pas illogique alors de les présenter comme un décor sur un mur, plutôt que comme une huile sur toile. Mais ceci est un autre débat.

\section{AUTEUR}

\section{MARIE-LAURE BERNADAC}

marie-laure.bernadac@louvre.fr 